\title{
Angiotensin II upregulates cyclophilin A by enhancing ROS production in rat cardiomyocytes
}

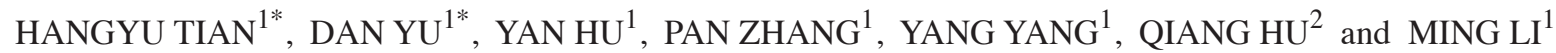 \\ ${ }^{1}$ Key Laboratory for Animal Genetic Diversity and Evolution of High Education in Yunnan Province, \\ School of Life Sciences, Yunnan University, Kunming, Yunnan 650091; ${ }^{2}$ Department of Emergency Medicine, \\ The Second Affiliated Hospital, Kunming Medical University, Kunming, Yunnan 650101, P.R. China
}

Received October 27, 2017; Accepted June 15, 2018

DOI: $10.3892 / \mathrm{mmr} .2018 .9448$

\begin{abstract}
Angiotensin II (Ang II) is a principal molecule of the renin-angiotensin system, which promotes hypertrophy and fibrosis. It has been demonstrated that Ang II upregulates the expression of cyclophilin A (CypA), which is a potential myocardial hypertrophy factor. However, the mechanisms by which Ang II induces the expression of CypA in cardiomyocytes remain unclear. In the present study, reactive oxygen species (ROS) were detected by fluorescence microscopy, and western blot analysis and ELISA were used to measure CypA expression. It was identified that Ang II enhanced the production of ROS in rat cardiomyocytes. ROS, in turn, promoted CypA expression and secretion. Notably, the action of Ang II was primarily dependent on the angiotensin type 2 receptor $\left(\mathrm{AT}_{2} \mathrm{R}\right)$, not the type 1 receptor. These results provided an insight into the role of the $\mathrm{AT}_{2} \mathrm{R}$ signaling pathway in Ang II-induced myocardial hypertrophy.
\end{abstract}

\section{Introduction}

Cardiac remodeling is associated with pathological alterations to the heart that are caused by myocardial infarction and hypertension, primarily via cardiomyocyte and myocardial

Correspondence to: Dr Ming Li, Key Laboratory for Animal Genetic Diversity and Evolution of High Education in Yunnan Province, School of Life Sciences, Yunnan University, 2 Cuihu Bei Road, Kunming, Yunnan 650091, P.R. China

E-mail: leeming@ynu.edu.cn

Dr Qiang Hu, Department of Emergency Medicine, The Second Affiliated Hospital, Kunming Medical University, 1 Mayuan Street, Kunming, Yunnan 650101, P.R. China

E-mail: yjmmt@126.com

${ }^{*}$ Contributed equally

Abbreviations: ROS, reactive oxygen species; Ang II, angiotensin II; CypA, cyclophilin A; AT1R, angiotensin type 1 receptor; AT2R, angiotensin type 2 receptor; GEE, glutathione ethyl ester; AAA, abdominal aortic aneurysm

Key words: Ang II, CypA, ROS, AT2R interstitial remodeling (1). Cardiomyocyte remodeling is characterized by compensatory hypertrophy, cell apoptosis and necrosis. Myocardial interstitial remodeling primarily refers to alterations in the extracellular matrix of cardiomyocytes, including fibroblast proliferation and fibrosis $(2,3)$. The renin-angiotensin system (RAS) has been identified to serve an important role in cardiac remodeling. Angiotensin II (Ang II) is a principal functional molecule in RAS, which is involved in myocardial fibrosis and accelerated myocardial remodeling (4-6).

Cyclophilin A (CypA), which was initially discovered in 1984 , is the primary target molecule of the immunosuppressant cyclosporine A (CsA) (7). CypA is involved in numerous biological activities, including protein folding $(8,9)$, inflammation (10-13), immunosuppression (14,15), apoptosis (16-19), and viral infection and replication $(20,21)$. A previous study conducted by Venkatesan et al (22) demonstrated that CypA is able to promote myocardial hypertrophy and exacerbate the severity of Ang II-induced myocardial hypertrophy. In rats, CsA effectively blocks or alleviates Ang II-induced myocardial hypertrophy by binding with CypA to form a dimer complex and inhibiting Ang II activity by binding to calcineurin $(1,22)$. Collectively, these results indicate that CypA is involved in Ang II-induced myocardial hypertrophy.

Reactive oxygen species (ROS) are active oxygen-containing compounds that are generated during biological aerobic metabolism. A series of responses, including cell proliferation, differentiation, migration, injury, matrix remodeling, apoptosis and necrosis, result from ROS production. ROS participate in signal transduction processes that control gene expression, cell growth and apoptosis (23-25). Furthermore, increased ROS production is considered to be a mechanism underlying Ang II-induced myocardial hypertrophy (26); however, the signaling pathways leading to Ang II-induced ROS production are not well understood (27).

Ang II is primarily recognized by two principal receptors in the cell membrane: Angiotensin type 1 receptor $\left(\mathrm{AT}_{1} \mathrm{R}\right)$ and angiotensin type 2 receptor $\left(\mathrm{AT}_{2} \mathrm{R}\right)$. Previously, Satoh et al demonstrated that, during the Ang II-induced formation of abdominal aortic aneurysm (AAA), CypA synergistically elevates ROS production (28). In the present study, the aim was to explore the mechanism of Ang II-induced myocardial hypertrophy. The results demonstrated that Ang II increased 
ROS production via the $\mathrm{AT}_{2} \mathrm{R}$ pathway in rat cardiomyocytes. ROS production, in turn, promoted CypA expression and secretion. These results suggested that ROS may serve an important role in the upregulation of CypA by Ang II.

\section{Materials and methods}

Rat cardiomyocyte culturing. Dulbecco's modified Eagle's medium (Thermo Fisher Scientific, Inc., Waltham, MA, USA) containing 10\% fetal bovine serum (cat. no. FSP500; Shanghai ExCell Biology, Inc., Shanghai, China) and F12 factor (HyClone; GE Healthcare, Chicago, IL, USA) was used to culture $\mathrm{H} 9 \mathrm{C} 2$ rat cardiomyocytes, which were provided by the State Key Laboratory of Natural Resource Conservation and Utilization (Kunming, China) in a $25-\mathrm{ml}$ filtered cell culture flask in an incubator containing $5 \% \mathrm{CO}_{2}$ at $37^{\circ} \mathrm{C}$. The cultures were observed daily and culture medium was changed every 2-3 days. When the cells had reached $\sim 80 \%$ confluence, the cells were washed three times in PBS and treated with $0.25 \%$ trypsin (HyClone; GE Healthcare) for digestion and passaging. The present study used the second and third passages of $\mathrm{H} 9 \mathrm{C} 2$ cells for experimentation.

ROS detection. H9C2 cells in the logarithmic growth phase were seeded at a density of $1 \times 10^{5}$ cells/well in 12-well plates and cultured at $37^{\circ} \mathrm{C}$ in $5 \% \mathrm{CO}_{2}$. Following cell adhesion, cells were subjected to different treatments: i) untreated; ii) 24-h incubation with $0.1 \mu \mathrm{M}$ Ang II (cat. no. A9290; Beijing Solarbio Science \& Technology Co., Ltd., Beijing, China); iii) 24-h incubation with $3 \mathrm{mM}$ glutathione ethyl ester (GEE; cat. no. 14953; Cayman Chemical Company, Ann Arbor, MI, USA) and $0.1 \mu \mathrm{M}$ Ang II; iv) 24-h incubation with $0.4 \mathrm{mM}$ butyrate (cat. no. B110438; Shanghai Aladdin Bio-Chem Technology Co., Ltd., Shanghai, China); v) pre-incubation with $10 \mu \mathrm{M}$ Valsartan (cat. no. MB1341-S; Dalian Meilun Biotech Co., Ltd., Dalian, China) for $1 \mathrm{~h}$, followed by 24-h incubation with $0.1 \mu \mathrm{M}$ Ang II; vi) 1-h pre-incubation with 1 mM PD123319 (cat. no. MB5078; Dalian Meilun Biotech Co., Ltd.), followed by $24-\mathrm{h}$ incubation with $0.1 \mu \mathrm{M}$ Ang II. Subsequently, the $\mathrm{H} 9 \mathrm{C} 2$ cells were treated with $50 \mu \mathrm{M}$ BES- $\mathrm{H}_{2} \mathrm{O}_{2}$-Ac (Wako Pure Chemical Industries, Ltd., Osaka, Japan) for $1 \mathrm{~h}$ prior to measuring the fluorescence intensity by fluorescence microscopy $(29,30)$.

Western blot analysis. $\mathrm{H} 9 \mathrm{C} 2$ cells in the logarithmic growth phase were used to seed a density of $2 \times 10^{5}$ cells into each well of a 6 -well plate. Following cell adhesion, cells were treated as described above. After the 24-h treatment, the $\mathrm{H} 9 \mathrm{C} 2$ cells were washed with PBS and harvested to extract total protein using radioimmunoprecipitation lysis buffer (Beijing Solarbio Science \& Technology Co., Ltd.). A bicinchoninic acid assay was used to quantify total protein concentration. Total protein $(50 \mu \mathrm{g})$ was loaded into each lane of an SDS-PAGE gel (10\% separation gel, $4 \%$ stacking gel), and the proteins were separated by electrophoresis at $90 \mathrm{~V}$ for $100 \mathrm{~min}$. Subsequently, proteins were transferred to nitrocellulose membranes at $300 \mathrm{~mA}$ for $100 \mathrm{~min}$, and the membranes were blocked with $4 \%$ skim milk at room temperature for $1 \mathrm{~h}$. After washing two times with PBS plus $0.05 \%$ Tween-20 (PBST; $5 \mathrm{~min} /$ wash), the membranes were incubated with
CypA-specific antibody, which was a rabbit polyclonal antibody generated for CypA, cloned from Microplitis bicoloratus hemocytes and expressed in Escherichia coli using the pET-32a-CypA plasmid (1:2,000; Bioworld Technology, Inc., Nanjing, China) and $\beta$-actin-specific antibody $(1: 2,000$; cat. no. BS6007M; Bioworld Technology, Inc.) at $4^{\circ} \mathrm{C}$ overnight with agitation. After washing with PBST twice, the membranes were incubated with Alexa Fluor ${ }^{\circledR} 568$ Goat Anti-Rabbit Immunoglobulin $\mathrm{G}$ (Heavy+Light) secondary antibody (1:2,000; cat. no. A0208; Beyotime Institute of Biotechnology, Haimen, China) at room temperature for $1 \mathrm{~h}$, followed by two washes with PBST for $10 \mathrm{~min}$. The protein bands were visualized with enhanced chemiluminescent reagent (Tanon Science and Technology Co., Ltd., Shanghai, China) using a Tanon 5200 imager (Tanon Science and Technology Co., Ltd.). Semi-quantification of the bands was performed using ImageJ software version 1.46r (National Institutes of Health, Bethesda, MD, USA), with the intensity values normalized to the corresponding $\beta$-actin band.

ELISA measurement of CypA expression in the culture medium. $\mathrm{H} 9 \mathrm{C} 2$ cells in the logarithmic growth phase were used to seed a density of $2 \times 10^{5}$ cells into each well of a 6 -well plate. Following cell adhesion, cells were treated as described above. The cell culture supernatant was collected in a $1.5-\mathrm{ml}$ sterile centrifuge tube and centrifuged at $800 \mathrm{x}$ g for $20 \mathrm{~min}$ at room temperature. The supernatant was transferred to a new 1.5-ml centrifuge tube and CypA protein content was measured using ELISA (cat. no. HZ-EL-R0298c; eBioscience; Thermo Fisher Scientific, Inc.), according to the manufacturer's protocol.

Statistical analysis. All experiments were repeated three times and the data are presented as the means \pm standard deviation. The green fluorescence signals of ROS were randomly collected and analyzed from three fields of vision using ImageJ software version 1.46r (National Institutes of Health). GraphPad Prism 6 (GraphPad Software, Inc., La Jolla, CA, USA) software was used for statistical analysis of all data in the present study. Statistical differences between the groups were analyzed using one-way analysis of variance followed by Tukey's post hoc test. $\mathrm{P}<0.05$ was considered to indicate a statistically significant difference.

\section{Results}

Ang II upregulates CypA in rat cardiomyocytes. Untreated mock $\mathrm{H} 9 \mathrm{C} 2$ cells exhibited relatively low CypA expression compared with the cells treated with Ang II. After a 24-h incubation with $0.1 \mu \mathrm{M}$ Ang II, H9C2 cells exhibited significantly upregulated CypA expression and increased CypA content in the supernatant $(\mathrm{P}<0.05 ;$ Fig. 1). These data suggested that Ang II may promote the expression and secretion of CypA in rat cardiomyocytes.

Ang II upregulates CypA via ROS. After a 24-h treatment with $0.1 \mu \mathrm{M}$ Ang II, ROS production was significantly increased in $\mathrm{H} 9 \mathrm{C} 2$ cells $(\mathrm{P}<0.05$; Fig. $2 \mathrm{~A}$ and $\mathrm{B})$. When ROS production was inhibited by treatment of $\mathrm{H} 9 \mathrm{C} 2$ cells with $3 \mathrm{mM}$ of the antioxidant glutathione ethyl ester, the 

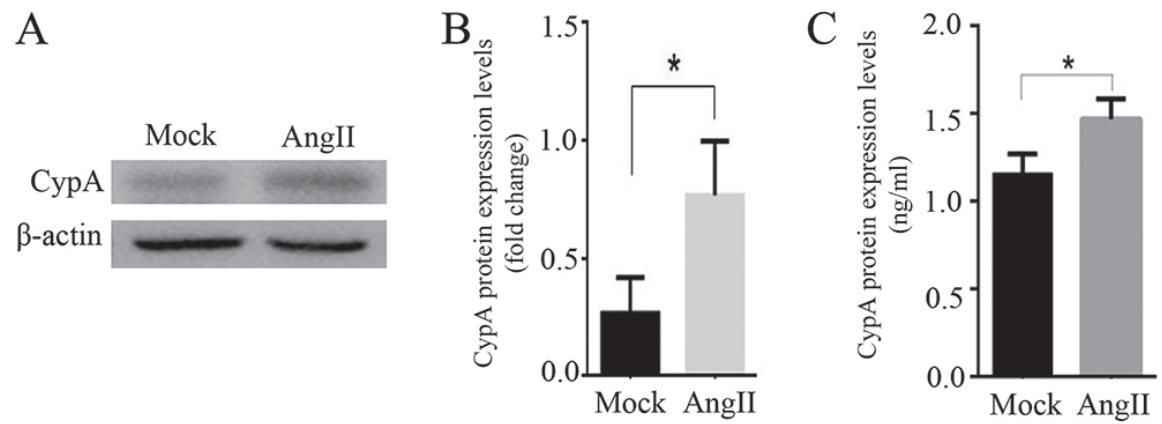

Figure 1. Ang II upregulates CypA in rat cardiomyocytes. (A) Western blotting and (B) semi-quantification analysis demonstrated that CypA expression was stimulated by Ang II in cultured H9C2 cells. (C) Ang II increased CypA content in the supernatant of cultured H9C2 cells. n=3/group. *P<0.05. Ang II, angiotensin II; CypA, cyclophilin A.
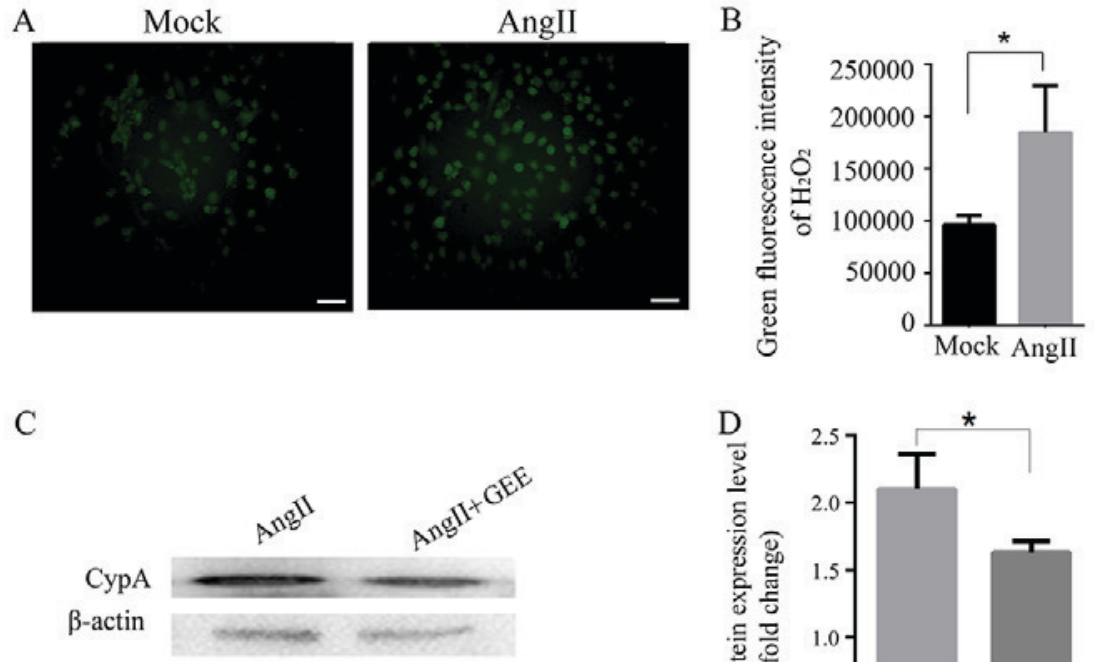

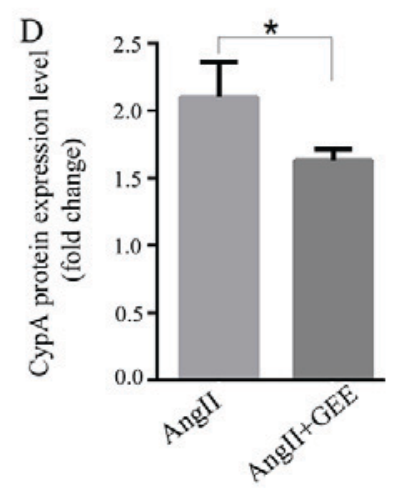

Figure 2. Ang II upregulates CypA via ROS. (A) Representative images and (B) analysis demonstrating that Ang II increased ROS production in cultured H9C2 cells. n=3/group. Green fluorescence represents ROS; scale bar, $50 \mu \mathrm{m}$. (C) Western blotting and (D) semi-quantification analysis demonstrated that Ang II-induced CypA expression was inhibited by GEE, a ROS inhibitor. * $\mathrm{P}<0.05$. Ang II, angiotensin II; CypA, cyclophilin A; GEE, glutathione ethyl ester; ROS, reactive oxygen species.

expression of CypA were significantly decreased $(\mathrm{P}<0.05$; Fig. 2C and D). These results suggested that Ang II may upregulate CypA by enhancing ROS production in rat cardiomyocytes.

Increased ROS induce the expression of CypA in rat cardiomyocytes. Butyrate, an oxidant, promotes ROS production in numerous types of cells $(31,32)$. In order to validate the role of ROS in upregulating CypA expression, $0.4 \mathrm{mM}$ butyrate was added to cells to stimulate ROS production. A ROS fluorescence probe (BES- $\mathrm{H}_{2} \mathrm{O}_{2}$-Ac) was used to evaluate ROS production over the course of $1 \mathrm{~h}$ under a fluorescence microscope. As hypothesized, ROS production was increased in $\mathrm{H} 9 \mathrm{C} 2$ cells following treatment with $0.4 \mathrm{mM}$ butyrate compared with in the control group $(\mathrm{P}<0.05$; Fig. $3 \mathrm{~A}$ and $\mathrm{B})$. Under butyrate stimulation, CypA in H9C2 cells and its content in the supernatant were significantly upregulated
$(\mathrm{P}<0.05$; Fig. 3C-E). These results indicated that increased ROS levels may promote the expression and secretion of CypA in rat cardiomyocytes.

Ang II increases ROS production via $A T_{2} R$. Ang II activates at least two pharmacologically distinct receptors, $\mathrm{AT}_{1} \mathrm{R}$ and $\mathrm{AT}_{2} \mathrm{R}$. To investigate which receptor is involved in ROS production, Valsartan and PD123319, which are antagonists of $\mathrm{AT}_{1} \mathrm{R}$ and $\mathrm{AT}_{2} \mathrm{R}$, respectively, were used in the present study. H9C2 cells were pre-incubated with $10 \mu \mathrm{M}$ Valsartan or $1 \mathrm{mM}$ PD123319 for $1 \mathrm{~h}$ prior to stimulation with $0.1 \mu \mathrm{M}$ Ang II. The results indicated that PD123319 significantly inhibited Ang II-induced ROS production ( $\mathrm{P}<0.001$; Fig. 4A and B). Valsartan additionally inhibited Ang II-induced ROS production; however, this effect was not statistically significant ( $\mathrm{P}=0.0637$; Fig. 4A and B). Notably, PD123319 significantly suppressed the CypA expression in $\mathrm{H} 9 \mathrm{C} 2$ cells $(\mathrm{P}<0.05$; 
A

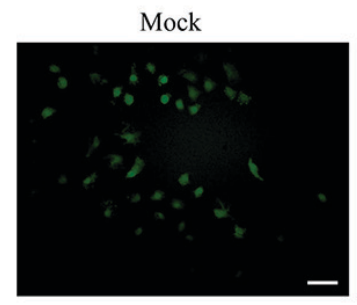

$\mathrm{C}$

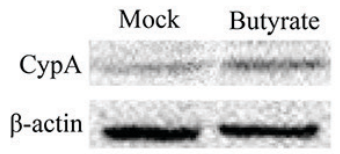

$\mathrm{D}$
Butyrate
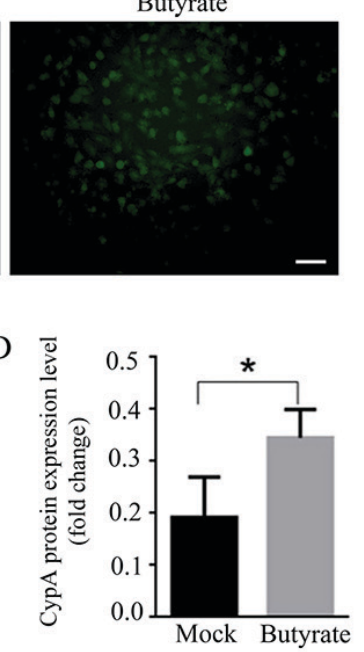

$\mathrm{B}$

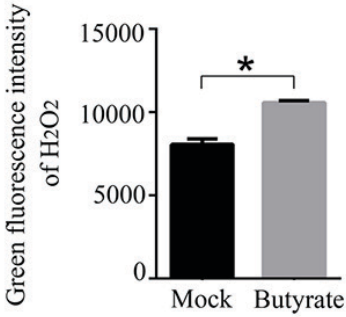

$\mathrm{E}$

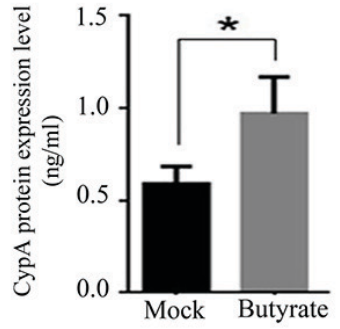

Figure 3. Elevated ROS induce the expression of CypA. (A) Representative images and (B) analysis demonstrating that butyrate promoted ROS production in cultured $\mathrm{H} 9 \mathrm{C} 2$ cells. $\mathrm{n}=3$ group; scale bar, $50 \mu \mathrm{m}$. (C) Western blotting and (D) semi-quantification analysis of CypA expression in H9C2 cells following treatment with butyrate. (E) CypA content analysis in the supernatant of $\mathrm{H} 9 \mathrm{C} 2$ cells following treatment with butyrate. "P<0.05. CypA, cyclophilin A; ROS, reactive oxygen species.

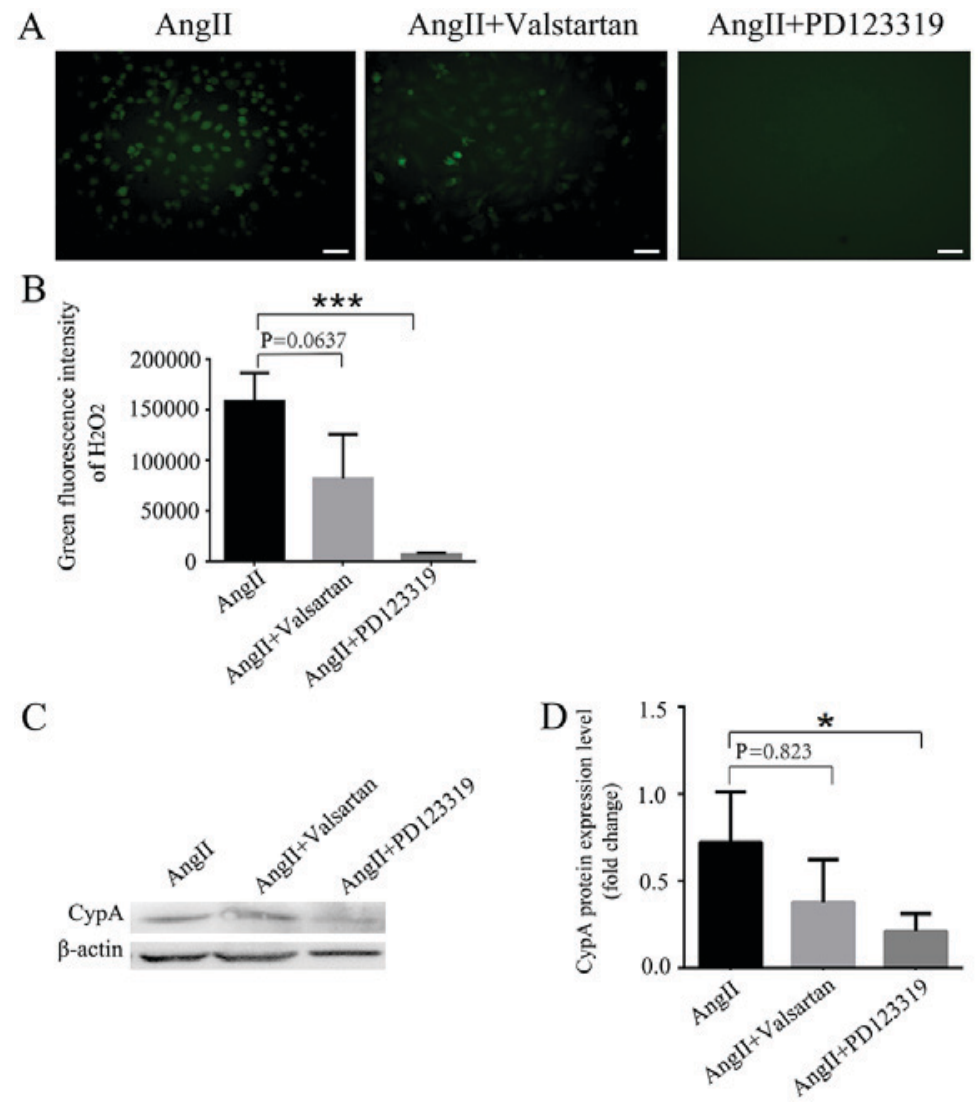

Figure 4. Ang II enhances ROS production via $\mathrm{AT}_{2} \mathrm{R}$. (A) Representative images and (B) analysis of ROS production in Ang II-induced H9C2 cells pretreated with Valsartan (angiotensin receptor 1 antagonist) and PD123319 (AT ${ }_{2} \mathrm{R}$ antagonist). Scale bar, $50 \mu \mathrm{m}$. PD123319 significantly inhibited Ang II-induced ROS production. $\mathrm{n}=3$ /group. (C) Western blotting and (D) semi-quantification analysis of CypA expression in Ang II-induced H9C2 cells pretreated with Valsartan and PD123319. PD123319 significantly suppressed Ang II-induced CypA expression in H9C2 cells. $n=3 /$ group. ${ }^{*} \mathrm{P}<0.05,{ }^{* * *} \mathrm{P}<0.01$. Ang II, angiotensin II; AT 2 , angiotensin receptor 2; CypA, cyclophilin A; ROS, reactive oxygen species.

Fig. 4C and D). Conversely, Valsartan did not significantly decrease CypA expression ( $\mathrm{P}=0.823)$. These results suggested that Ang II upregulated CypA by enhancing ROS production primarily via the $\mathrm{AT}_{2} \mathrm{R}$ pathway in rat cardiomyocytes.

\section{Discussion}

Accumulating evidence indicates that Ang II serves an important role in myocardial fibrosis and hypertrophy, which 


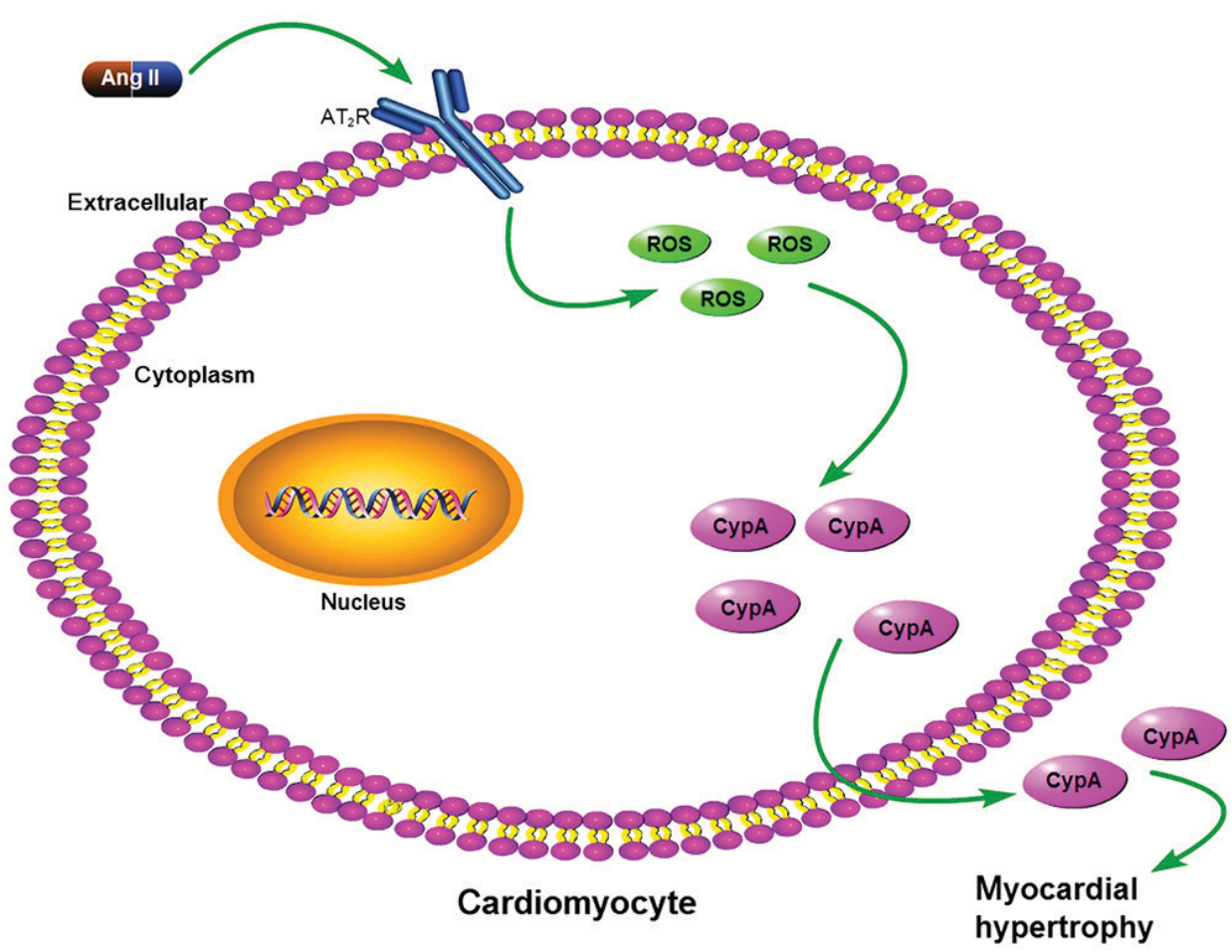

Figure 5. Proposed mechanism of how Ang II upregulates CypA. Ang II increased ROS levels via $\mathrm{AT}_{2} \mathrm{R}$ in cardiomyocytes; increased ROS, in turn, promoted CypA expression and secretion. Ang II, angiotensin II; $\mathrm{AT}_{2} \mathrm{R}$, angiotensin type 2 receptor; CypA, cyclophilin A; ROS, reactive oxygen species.

are important features in cardiac remodeling $(6,27,33)$. Satoh et al (28) demonstrated that CypA serves a key role in Ang II-induced AAA formation using gene knockout animal models. AAA formation is a typical manifestation of vascular remodeling that is caused by atherosclerosis. These results suggested that CypA is likely to serve the same role in Ang II-induced cardiac remodeling; however, the mechanism underlying Ang II-induced CypA expression remains unclear. Previously, Seko et al (34) demonstrated that hypoxia followed by reoxygenation may promote CypA secretion in cultured rat cardiomyocytes. In the present study, it was identified that Ang II promoted CypA expression and secretion, in addition to ROS production in $\mathrm{H} 9 \mathrm{C} 2$ cells. These results suggested that CypA expression may be associated with ROS formation. Notably, when the formation of ROS was inhibited by the antioxidants GEE and PD123319, CypA expression was downregulated in Ang II-treated rat cardiomyocytes. Furthermore, it was identified that an increase in ROS formation induced by butyrate upregulated the expression of CypA. These results indicated that Ang II upregulated CypA in a ROS-dependent manner.

The biological activities of Ang II, a primary functional molecule of the RAS, are primarily mediated by its two G-protein-coupled receptors, $A_{1} R$ and $A_{2} R(35)$. $A_{1} T_{1} R$ is documented to mediate the majority of the biological functions of Ang II, whereas the roles of $\mathrm{AT}_{2} \mathrm{R}$ are considered to counterbalance the effects mediated by activation of $A T_{1} R$. Previous studies have demonstrated that long-term Ang II injection causes elevated blood pressure in mice, leading to myocardial hypertrophy, fibrosis and activation of $\mathrm{AT}_{1} \mathrm{R}(6,36)$. Ang II promotes cardiomyocyte proliferation and growth, collagen formation, extracellular matrix deposition and myocardial fibrosis via $\mathrm{AT}_{1} \mathrm{R}$ (37). Therefore,
Ang II is involved in cardiac remodeling through the $\mathrm{AT}_{1} \mathrm{R}$ pathway. However, $\mathrm{AT}_{2} \mathrm{R}$ expression is upregulated in pathological circumstances, including in heart failure, experimental cardiac hypertrophy, myocardial infarction and vascular injury $(38,39)$. In the present study, it was observed that Ang II induced ROS production primarily via $\mathrm{AT}_{2} \mathrm{R}$, which is consistent with results of Thakur et al (40); in their research, Ang II induced ROS production by NADPH oxidase 2 in endothelial cells. ROS, in turn, may promote CypA expression and secretion. Therefore, it may be suggested that $\mathrm{AT}_{2} \mathrm{R}$ exhibits its function in Ang II-mediated myocardial hypertrophy, at least in part, by upregulating CypA expression (Fig. 5).

Cardiac remodeling is associated with the apoptosis and necrosis of a large number of cardiomyocytes. In the present study, it was revealed that Ang II may promote CypA expression by stimulating ROS production. Satoh et al (28) suggested that CypA may increase ROS production in vascular tissue, and Seko et al (34), demonstrated that cultured rat cardiomyocytes are able to promote CypA secretion during reoxidation, thus indicating that during Ang II stimulation in rat cardiomyocytes the following cycle may be exhibited: Ang II binds to $\mathrm{AT}_{2} \mathrm{R}$ and upregulates CypA expression and secretion by promoting ROS production, in turn, CypA may increase ROS production. The positive association between CypA expression and ROS production is consistent with the results of Perrucci et al (41); during the course of CypA-promoted angiogenesis, low CypA concentrations promote the proliferation of bone marrow-derived cluster of differentiation (CD) $117^{+}$cells, whereas high CypA concentrations stimulate $\mathrm{CD} 117^{+}$cell death. Therefore, it is reasonable to speculate that, during Ang II-induced cardiac remodeling, the positive association between CypA and ROS may result in CypA and ROS accumulation. A continuous increase of these 
two molecules promotes cell death, thereby accelerating the processes of cardiac reconstruction. An in vitro study suggested that CypA is an oxidative stress-inducing factor, whereas in vascular smooth muscle cells, ROS activates a vesicle-containing pathway that leads to CypA secretion; this process requires the involvement of Rho GTPases (including transforming protein RhoA, cell division control protein 42 homolog and Ras-related C3 botulinum toxin substrate 1) (42).

In conclusion, to the best of our knowledge, the present study is the first to demonstrate that Ang II may induce CypA expression and secretion by enhancing ROS production. In addition, Ang II promoted ROS formation primarily via the $\mathrm{AT}_{2} \mathrm{R}$ signaling pathway in rat cardiomyocytes. These results provided further insight into the role of $\mathrm{AT}_{2} \mathrm{R}$ signaling in Ang II-induced myocardial hypertrophy and may aid in the development of novel therapeutic strategies for treatment of cardiovascular disease.

\section{Acknowledgements}

The authors would like to thank Professor Chenggang Zou from the State Key Laboratory of Natural Resource Conservation and Utilization (Kunming, China) for providing the H9C2 cells. The authors are grateful to Dr Zhiwen Zhu from Tsinghua-Peking Center for Life Sciences, School of Life Sciences, Tsinghua University (Beijing, China) for discussions regarding the present study.

\section{Funding}

The present study was supported by grants from the National Natural Science Foundation of China (grant no. 31360454 and 31560528) and a grant (grant no. 2012FB120) from the Yunnan Department of Science and Technology.

\section{Availability of data and materials}

The analyzed data sets generated during the present study are available from the corresponding author upon reasonable request.

\section{Authors' contributions}

ML and QH conceived the present study. HT and DY performed the experiments and collected and analyzed the data. HT and ML analyzed the data, wrote the manuscript and discussed the paper. YH and PZ performed cell culture experiments. YY provided guidance on fluorescence microscopy and analyzed the fluorescence data.

\section{Ethics approval and consent to participate}

Not applicable.

\section{Patient consent for publication}

Not applicable.

\section{Competing interests}

The authors declare that they have no competing interests.

\section{References}

1. Satoh K, Shimokawa H and Berk BC: Cyclophilin A-promising new target in cardiovascular therapy. Circ J 74: 2249-2256, 2010.

2. Bernardo BC, Weeks KL, Pretorius L and McMullen JR: Molecular distinction between physiological and pathological cardiac hypertrophy: Experimental findings and therapeutic strategies. Pharmacol Ther 128: 191-227, 2010.

3. Diwan A and Dorn GW II: Decompensation of cardiac hypertrophy: Cellular mechanisms and novel therapeutic targets. Physiology (Bethesda) 22: 56-64, 2007.

4. Olson ER, Shamhart PE, Naugle JE and Meszaros JG: Angiotensin II-induced extracellular signal-regulated kinase $1 / 2$ activation is mediated by protein kinase Cdelta and intracellular calcium in adult rat cardiac fibroblasts. Hypertension 51: 704-711, 2008

5. Rosenkranz S: TGF-beta1 and angiotensin networking in cardiac remodeling. Cardiovasc Res 63: 423-432, 2004.

6. Matsumoto E, Sasaki S, Kinoshita H, Kito T, Ohta H, Konishi M, Kuwahara K, Nakao K and Itoh N: Angiotensin II-induced cardiac hypertrophy and fibrosis are promoted in mice lacking Fgf16. Genes Cells 18: 544-553, 2013.

7. Handschumacher R, Harding M, Rice J, Drugge R and Speicher D: Cyclophilin: A specific cytosolic binding protein for cyclosporin a. Science 226: 544-547, 1984.

8. Göthel S and Marahiel M: Peptidyl-prolyl cis-trans isomerases, a superfamily of ubiquitous folding catalysts. Cell Mol Life Sci 55: 423-436, 1999.

9. Ivery MT: Immunophilins: Switched on protein binding domains? Med Res Rev 20: 452-484, 2000.

10. Liu L, Li C, Cai C, Xiang J and Cao Z: Cyclophilin a (CypA) is associated with the inflammatory infiltration and alveolar bone destruction in an experimental periodontitis. Biochem Biophys Res Commun 391: 1000-1006, 2010.

11. Seizer P, Geisler T, Bigalke B, Schneider M,Klingel K, Kandolf R, Stellos K, Schreieck J, Gawaz M and May AE: EMMPRIN and its ligand cyclophilin a as novel diagnostic markers in inflammatory cardiomyopathy. Int J Cardiol 163: 299-304, 2013.

12. Seizer P, Klingel K, Sauter M, Westermann D, Ochmann C, Schönberger T, Schleicher R, Stellos K, Schmidt EM, Borst $\mathrm{O}$, et al: Cyclophilin a affects inflammation, virus elimination and myocardial fibrosis in coxsackievirus B3-induced myocarditis. J Mol Cell Cardiol 53: 6-14, 2012.

13. Takapoo M, Chamseddine AH, Bhalla RC and Miller FJ Jr: Glutathione peroxidase-deficient smooth muscle cells cause paracrine activation of normal smooth muscle cells via cyclophilin a. Vascul Pharmacol 55: 143-148, 2011.

14. Jabri B and Barreiro LB: Don't move: LRRK2 arrests NFAT in the cytoplasm. Nat Immunol 12: 1029-1030, 2011.

15. Li H, Pink MD, Murphy JG, Stein A, Dell'Acqua ML and Hogan PG: Balanced interactions of calcineurin with AKAP79 regulate $\mathrm{Ca} 2+-$ calcineurin-NFAT signaling. Nat Struct Mol Biol 19: 337-345, 2012.

16. Candé C, Vahsen N, Kouranti I, Schmitt E, Daugas E, Spahr C, Luban J, Kroemer RT, Giordanetto F, Garrido C, et al: AIF and cyclophilin a cooperate in apoptosis-associated chromatinolysis. Oncogene 23: 1514-1521, 2004.

17. Tanaka H, Shimazaki H, Kimura M, Izuta H, Tsuruma K, Shimazawa M, Hara H: Apoptosis-inducing factor and cyclophilin a cotranslocate to the motor neuronal nuclei in amyotrophic lateral sclerosis model mice. CNS Neurosci Ther 17: 294-304, 2011.

18. Zhu C, Wang X, Deinum J, Huang Z, Gao J, Modjtahedi N, Neagu MR, Nilsson M, Eriksson PS, Hagberg $\mathrm{H}$, et al: Cyclophilin a participates in the nuclear translocation of apoptosis-inducing factor in neurons after cerebral hypoxia-ischemia. J Exp Med 204: 1741-1748, 2007.

19. Piao CS, Loane DJ, Stoica BA, Li S, Hanscom M, Cabatbat R, Blomgren K and Faden AI: Combined inhibition of cell death induced by apoptosis inducing factor and caspases provides additive neuroprotection in experimental traumatic brain injury. Neurobiol Dis 46: 745-758, 2012.

20. He H, Zhou D, Fan W, Fu X, Zhang J, Shen Z, Li J, Li J and Wu Y: Cyclophilin a inhibits rotavirus replication by facilitating host IFN-I production. Biochem Biophys Res Commun 422: 664-669, 2012.

21. Zhou D, Mei Q, Li J and He H: Cyclophilin a and viral infections. Biochem Biophys Res Commun 424: 647-650, 2012. 
22. Venkatesan B, Valente AJ,Prabhu SD, Shanmugam P, Delafontaine P and Chandrasekar B: EMMPRIN activates multiple transcription factors in cardiomyocytes, and induces interleukin-18 expression via Rac1-dependent PI3K/Akt/IKK/NF-kappaB andMKK7/JNK/AP-1 signaling. J Mol Cell Cardiol 49: 655-663, 2010.

23. de Gasparo M and Siragy HM: The AT2 receptor: Fact, fancy and fantasy. Regul Pept 81: 11-24, 1999.

24. Ma Y, Kong L, Qi S and Wang D: Atorvastatin blocks increased 1-type $\mathrm{Ca} 2+$ current and cell injury elicited by angiotensin II via inhibiting oxide stress. Acta Biochim Biophys Sin (Shanghai) 48 : 378-384, 2016.

25. Martens GA, Cai Y, Hinke S, Stangé G, Van de Casteele M and Pipeleers D: Glucose suppresses superoxide generation in metabolically responsive pancreatic beta cells. J Biol Chem 280 : 20389-20396, 2005

26. Perrotta I and Aquila S: The role of oxidative stress and autophagy in atherosclerosis. Oxid Med Cell Longev 2015: 130315, 2015.

27. Mehta PK and Griendling KK: Angiotensin II cell signaling: Physiological and pathological effects in the cardiovascular system. Am J Physiol Cell Physiol 292: C82-C97, 2007.

28. Satoh K, Nigro P, Matoba T, O'Dell MR, Cui Z, Shi X, Mohan A, Yan C, Abe J, Illig KA and Berk BC: Cyclophilin a enhances vascular oxidative stress and the development of angiotensin II-induced aortic aneurysms. Nat Med 15: 649-656, 2009.

29. Maeda H, Yamamoto K, Kohno I, Hafsi L, Itoh N, Nakagawa S, Kanagawa N, Suzuki K and Uno T: Design of a practical fluorescent probe for superoxide based on protection-deprotection chemistry of fluoresceins with benzenesulfonyl protecting groups. Chemistry 13: 1946-1954, 2007

30. Maeda H, Yamamoto K, Nomura Y, Kohno I, Hafsi L, Ueda N, Yoshida S, Fukuda M, Fukuyasu Y, Yamauchi Y and Itoh N: A design of fluorescent probes for superoxide based on a nonredox mechanism. J Am Chem Soc 127: 68-69, 2005.

31. Chang MC, Tsai YL, Chen YW, Chan CP, Huang CF, Lan WC, Lin CC, Lan WH and Jeng JH: Butyrate induces reactive oxygen species production and affects cell cycle progression in human gingival fibroblasts. J Periodontal Res 48: 66-73, 2013.

32. Liu Q, Shimoyama T, Suzuki K, Umeda T, Nakaji S and Sugawara K: Effect of sodium butyrate on reactive oxygen species generation by human neutrophils. Scand J Gastroenterol 36: 744-750, 2001.

33. Wang LP, Wang Y, Zhao LM, Li GR and Deng XL: Angiotensin II upregulates $\mathrm{K}(\mathrm{Ca}) 3.1$ channels and stimulates cell proliferation in rat cardiac fibroblasts. Biochem Pharmacol 85: 1486-1494, 2013.
34. Seko Y, Fujimura T, Taka H, Mineki R, Murayama K and Nagai R: Hypoxia followed by reoxygenation induces secretion of cyclophilin a from cultured rat cardiac myocytes. Biochem Biophys Res Commun 317: 162-168, 2004.

35. Savoia C, Burger D, Nishigaki N, Montezano A and Touyz RM: Angiotensin II and the vascular phenotype in hypertension. Expert Rev Mol Med 13: e11, 2011.

36. Mercure C, Yogi A, Callera GE, Aranha AB, Bader M,Ferreira AJ, Santos RA, Walther T, Touyz RM and Reudelhuber TL: Angiotensin(1-7) blunts hypertensive cardiac remodeling by a direct effect on the heart. Circ Res 103: 1319-1326, 2008.

37. Vinturache AE and Smith FG: Angiotensin type 1 and type 2 receptors during ontogeny: Cardiovascular and renal effects. Vascul Pharmacol 63: 145-154, 2014

38. Lopez JJ, Lorell BH, Ingelfinger JR, Weinberg EO, Schunkert H, Diamant D and Tang SS: Distribution and function of cardiac angiotensin AT1- and AT2-receptor subtypes in hypertrophied rat hearts. Am J Physiol 267: H844-H852, 1994.

39. Ohkubo N, Matsubara H, Nozawa Y, Mori Y, Murasawa S, Kijima K, Maruyama K, Masaki H, Tsutumi Y, Shibazaki Y, et al: Angiotensin type 2 receptors are reexpressed by cardiac fibroblasts from failing myopathic hamster hearts and inhibit cell growth and fibrillar collagen metabolism. Circulation 96: 3954-3962, 1997.

40. Thakur S, Du J, Hourani S, Ledent C and Li JM: Inactivation of adenosine A2A receptor attenuates basal and angiotensin II-induced ROS production by Nox 2 in endothelial cells. J Biol Chem 285: 40104-40113, 2010.

41. Perrucci GL, Straino S, Corlianò M, Scopece A, Napolitano M, Berk BC, Lombardi F, Pompilio G, Capogrossi MC and Nigro P: Cyclophilin a modulates bone marrow-derived CD117(+) cells and enhances ischemia-induced angiogenesis via the SDF-1/CXCR4 axis. Int J Cardiol 212: 324-335, 2016.

42. Nagai T, Anzai T, Kaneko H, Mano Y, Anzai A, Maekawa Y, Takahashi T, Meguro T, Yoshikawa T and Fukuda K: C-reactive protein overexpression exacerbates pressure overload-induced cardiac remodeling through enhanced inflammatory response. Hypertension 57: 208-215, 2011.

This work is licensed under a Creative Commons Attribution-NonCommercial-NoDerivatives 4.0 International (CC BY-NC-ND 4.0) License. 